Copyright @ 2008 IEEE.

Reprinted from IEEE Transactions on Power Delivery, 2008; 23

(4):2417-2425

This material is posted here with permission of the IEEE. Such permission of the IEEE does not in any way imply IEEE endorsement of any of the University of Adelaide's products or services. Internal or personal use of this material is permitted. However, permission to reprint/republish this material for advertising or promotional purposes or for creating new collective works for resale or redistribution must be obtained from the IEEE by writing to pubs-permissions@ieee.org.

By choosing to view this document, you agree to all provisions of the copyright laws protecting it. 


\title{
Automatic Classification and Characterization of Power Quality Events
}

\author{
Ameen M. Gargoom, Student Member, IEEE, Nesimi Ertugrul, Member, IEEE, and Wen. L. Soong, Member, IEEE
}

\begin{abstract}
This paper presents a new technique for automatic monitoring of power quality events, which is based on the multiresolution S-transform and Parseval's theorem. In the proposed technique, the $S$-transform is used to produce instantaneous frequency vectors of the signals, and then the energies of these vectors, based on the Parseval's theorem, are utilized for automatically monitoring and classification of power quality events. The advantage of the proposed algorithm is its ability to distinguish different power quality classes easily. In addition, the magnitude, duration, and frequency content of the disturbances can be accurately identified in order to characterize the disturbances. The paper provides the theoretical background of the technique and presents a wide range of analyses to demonstrate its effectiveness.
\end{abstract}

Index Terms-Automatic classification, Parseval's theorem, power quality monitoring, S-transform.

\section{INTRODUCTION}

I $\mathrm{N}$ THE industrialized world, electric power systems have become polluted with unwanted variations in the voltage and current signals. This power quality issue is primarily due to continually increasing sources of disturbances that occur in interconnected power grids, which contain large numbers of power sources, transmission lines, transformers and loads. In addition, such systems are exposed to environmental disturbances like lighting strikes. Furthermore, nonlinear power electronic loads, such as converter-driven equipment (which varies from consumer electronics and computers, up to adjustable-speed drives), have become increasingly common in power systems. Although these converter-driven equipment are manufactured according to the appropriate standards, the wide utilization of such devices pollutes the power systems. If these unwanted variations in the voltage and current signals are not mitigated properly, they can lead to failures or malfunctions of the many sensitive loads connected to the same systems, which may be very costly for the end users.

As a result, power quality issues have become a major concern in the power industry, particularly, in this era of deregulation. An important step in understanding and hence improving the quality of the electric power is to extract sufficient information about the events that cause power quality issues. This is currently obtained by using a large number of recorders located through

Manuscript received August 14, 2006; revised October 5, 2007. First published June 27, 2008; current version published September 24, 2008. Paper no. TPWRD-00474-2006.

The authors are with the Department of Electrical and Electronic Engineering, University of Adelaide, Adelaide SA5005, Australia (e-mail: agargoom@eleceng.adelaide.edu.au).

Color versions of one or more of the figures in this paper are available online at http://ieeexplore.ieee.org.

Digital Object Identifier 10.1109/TPWRD.2008.923998 the power system. However, when we consider the continually growing number of monitors installed and the large amount of data that may be collected in a given power system, the manual inspection and extraction of information about the events is no longer a practical option in any monitoring process. Therefore, it is necessary to have automatic analysis tools, integrated with the monitoring systems that can be applied to large existing databases for automatic analysis and classification of power quality events. This information is important for identifying the cause of these events and selecting appropriate mitigation techniques.

A number of techniques have been investigated in the literature for the automatic classification of different types of power quality events. Some survey studies can be found in [1]-[3].

Among these techniques, the Fourier Transform (FT) is the most commonly used technique to obtain harmonic information about the signals monitored. However, using the FT alone is inadequate for feature extraction due to the transient nature of most power quality signals where time information is required for analyzing such signals.

To overcome the inadequacy of the Fourier transform (FT), the short-time Fourier transform (STFT) technique was adopted in [4] for detecting and characterizing power quality disturbances in the time-frequency domain. The signal studied was split into a set of output signals, which were obtained using bandpass filters centered at the harmonic frequencies. Although the STFT technique offered a partial solution for the absence of time information in the FT, it has also limitations due to its fixed window length, which has to be chosen prior to the analysis. This drawback is reflected in the achievable frequency resolution when analyzing nonstationary signals with both lowand high-frequency components [5].

In order to overcome the limitations of the Fourier-based algorithms, the wavelet transform (WT) technique has been utilized, and shown to be suitable for power quality analysis, specifically for nonstationary signals. Due to its ability to analyze signals at different frequency resolutions, the technique has been explored extensively in various studies as an alternative to the STFT, [6]-[10]. This research covered both the continuous wavelet transform (CWT) and the discrete wavelet transform (DWT).

The previous work on the CWT was mainly based on the visual detection of power quality events in the time-scale plane [6], [7], which is capable of handling noisy signals. However, the main disadvantage of the CWT is its redundancy of using a large number of scales resulting in a significant computational overhead.

To avoid the redundancy in the CWT technique, the wavelet multiresolution analysis (MRA) was adopted by a number of researchers. In [8]-[10], an algorithm based on the energies of the 

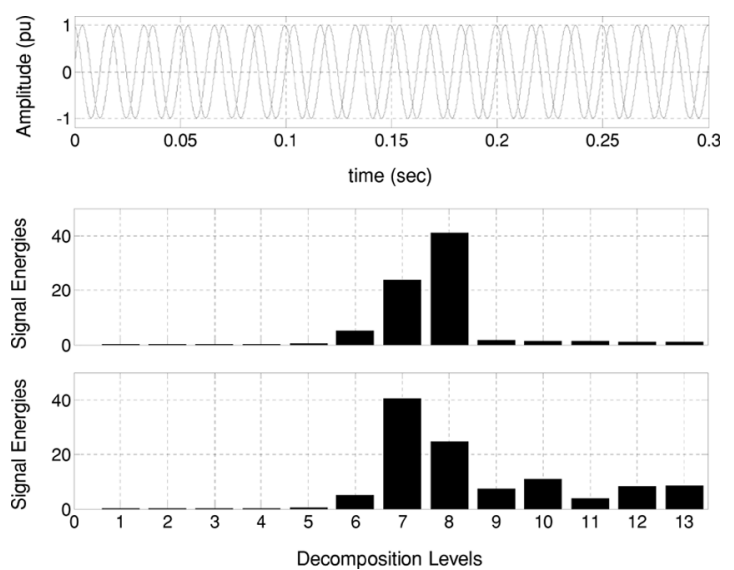

Fig. 1. Two pure sinewave signals with different phase shifts (top), and their decomposed signals energy distribution diagrams (middle and bottom).

decomposed signals from wavelet MRA was proposed to distinguish different classes of power quality events. Although the energies of the decomposed signals at different levels were shown to be sensitive to the type of disturbance and, thus, were used as distinctive features for the classification, the phase shifts of the signals studied were not considered despite their impact on the results [11]. This issue can be illustrated in Fig. 1, which shows two pure sine waves with different phase shifts (Fig. 1 top), and their energies at different levels based on MRA (Fig. 1 middle and bottom). It can be seen in this figure that, although the two analyzed signals are ideal sine waves, their energy distributions are different at the levels from 7 to 13 . These differences make it more difficult to distinguish changes in the energy distributions due to distorted signals as opposed to phase shifts in the signals being processed. Another issue related to wavelet analysis is its limited ability to classify disturbances that have more gradual changes, such as sags or swells events that start at the zero-crossings of the measured signal [12].

As an alternate to the wavelet-based techniques, the multiresolution S-transform technique (MRST) was presented in [13] as a new power quality signal processing technique. The MRST technique was used for classifying seven classes of power quality events based on three extracted features calculated from the minimum and maximum values of the S-transform matrix. However, these features were not able to provide additional information about the characteristics of disturbances.

Therefore, based on the above discussion, it was concluded that the previous studies were focused on the implementation of different signal processing techniques for only distinguishing, (classifying), different types of power quality events. However, for a detailed power quality analysis, it is expected that the adopted power quality techniques should be capable of both classifying the type of power quality events and identifying characteristics such as their magnitude, duration and frequency content.

To achieve this, the paper proposes a new algorithm that is based on the MRST technique and Parseval's Theorem for classifying and characterizing the power quality events. The mathematical background of the MRST and the S-Transform spectrum are discussed in the Sections II and III, respectively. The proposed automatic classification algorithm is explained

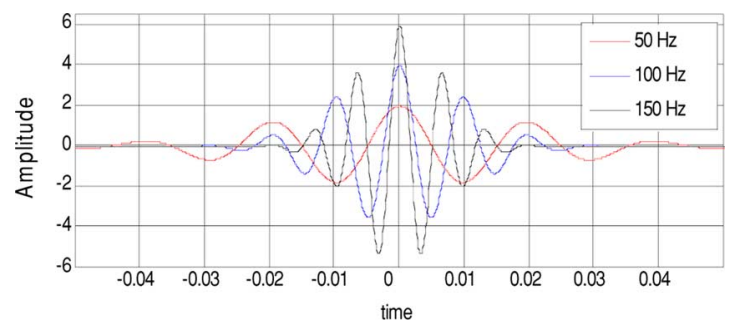

Fig. 2. Gaussian window function for ST at three different frequencies.

in Section IV. The application of the algorithm is given in Section V, and the paper draws its conclusions in Section VI.

\section{Multiresolution S-Transform}

The S-transform (ST) technique was introduced in [13] as an alternative to the STFT technique for localization of the time-frequency spectra. Such a technique can be considered conceptually as a hybrid of the STFT and the CWT techniques. The ST can provide time and frequency information like the STFT but it does this at different resolutions using a variable window length as in the CWT technique [5], [6].

The ST technique is derived from the CWT by modifying the phase of the window function (mother wavelet function) in the CWT. Therefore, for a continuous time signal, $v(t)$, the ST can be derived as in [13] as the CWT of the product of the target signal and a phase correction function $\exp (-j 2 \pi f \tau)$

$$
V_{\mathrm{ST}}(\tau, a, f)=\int_{-\infty}^{\infty} v(t) \cdot \psi(t-\tau, a) \cdot \exp (-j 2 \pi f \tau) \cdot d t
$$

where $V_{\mathrm{ST}}$ is the $\mathrm{ST}$ of the signal $v(t), \psi$ is the mother wavelet function, $f$ is the frequency, $a$ is the wavelet scale, and $j$ is equal to $\sqrt{ }-1$.

However, unlike the WT technique, the mother Wavelet (the window function) in the ST is chosen to be a function of the frequency content in the signal instead of the scale $a$. This is given as

$$
\psi(t, \sigma, f)=\frac{1}{\sigma \sqrt{2 \pi}} \exp \left(-t^{2} / 2 \sigma^{2}\right) \cdot \exp (-j 2 \pi f t)
$$

where $\sigma$ is a function that controls the length of the window function, and it is chosen to be proportional to the inverse of the frequency of the signal. Fig. 2 shows a plot of the above function at three different frequencies.

Thus, if (2) is substituted into (1), the ST can be given as

$$
V_{\mathrm{ST}}(\tau, \sigma, f)=\int_{-\infty}^{\infty} v(t) \cdot \frac{1}{\sigma \sqrt{2 \pi}} \cdot e^{\frac{-(t-\tau)^{2}}{2 \sigma^{2}}} \cdot e^{-j 2 \pi f t} \cdot d t .
$$

The $\sigma$ function in the above equation is proportional to the inverse of the frequency content of the signal and is defined in [13] as

$$
\sigma(f)=\frac{1}{c+b|f|}
$$

where $b$ and $c$ are constants, and $b \neq 0$. Note that, setting $b$ to zero, transforms the window function to the same window that 


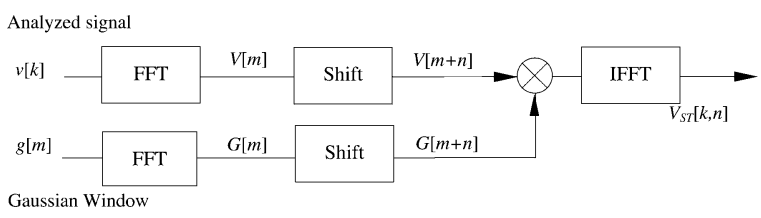

Fig. 3. Computation process of the ST.

is used in the STFT technique. For simplicity, $c$ can be set to zero, and thus the ST of the signal can be rewritten as

$$
V_{\mathrm{ST}}(\tau, f)=\int_{-\infty}^{\infty} v(t) \cdot \frac{|f|}{b \sqrt{2 \pi}} \cdot e^{\frac{-(t-\tau)^{2}}{2 \sigma^{2}}} \cdot e^{-j 2 \pi f t} \cdot d t
$$

The above equation represents a convolution of the continuous time series $v(t)$ with a frequency-dependant window function $|f| /(b \sqrt{ }(2 \pi)) \cdot \exp \left(-t^{2} f^{2} / 2\right)$ which is known as the Gaussian window function. Therefore, as the convolution process in the time domain is equivalent to a multiplication in the frequency domain, the ST can be calculated by multiplying the Fourier transform of the analyzed signal $v(t)$ and the Fourier transform of the Gaussian window function. The Fourier transform of the Gaussian function is calculated as

$$
G(f)=e^{\frac{-2 \pi^{2} \alpha^{2}}{f^{2}}}
$$

where $\alpha$ is a constant that is equal to $1 / b$ as defined in (4). The ST technique produces a multiresolution analysis as a bank of filters with a constant relative bandwidth [13].

It can be seen in (5) that, due to the term $\exp (-j 2 \pi f t)$, the $\mathrm{ST}$ is a complex transform. Therefore, the ST can be written as

$$
V_{\mathrm{ST}}(\tau, f)=S(\tau, f) \cdot e^{j \varphi(\tau, f)}
$$

where $S(\tau, f)$ and $\varphi(\tau, f)$ are the amplitude and phase of the ST spectrum, respectively.

In practical applications, where the captured signals are in discrete form, the discrete version of the ST technique is obtained by making $f$ equal to $n / N T$ and $\tau$ equal to $k T$

$$
V_{\mathrm{ST}}\left[k T, \frac{n}{N T}\right]=\sum_{m=0}^{N-1} V\left[\frac{m+n}{N T}\right] \cdot e^{-\frac{2 \pi^{2} m^{2} \alpha^{2}}{n^{2}}} \cdot e^{j \frac{2 \pi n}{N} k}
$$

where $N$ is the length of the analyzed signal, the indices $k, m$, and $n$ are equal to $0,1, \ldots N-1, T$ is the time interval between two consecutive samples, $V[(m+n) / N T]$ is the fast Fourier transform of the analyzed signal, and $j=\sqrt{ }-1$.

The computation process of the ST can be illustrated in a block diagram form as shown in Fig. 3. As shown in the figure, the analyzed signal and the ST Gaussian window are transformed into the frequency domain using the FFT technique, and both spectrums are localized around the required frequency. Then, the ST is obtained by multiplying both shifted spectrums and taking the inverse FFT of the product.

\section{S-TRANSFORM SPECTRUM}

The amplitude of the ST spectrum $(S[k T, n / N T])$ is defined by the absolute values of the complex matrix resulting from the ST technique, which is

$$
S\left[k T, \frac{n}{N T}\right]=\left|V_{\mathrm{ST}}\left[k T, \frac{n}{N T}\right]\right| .
$$

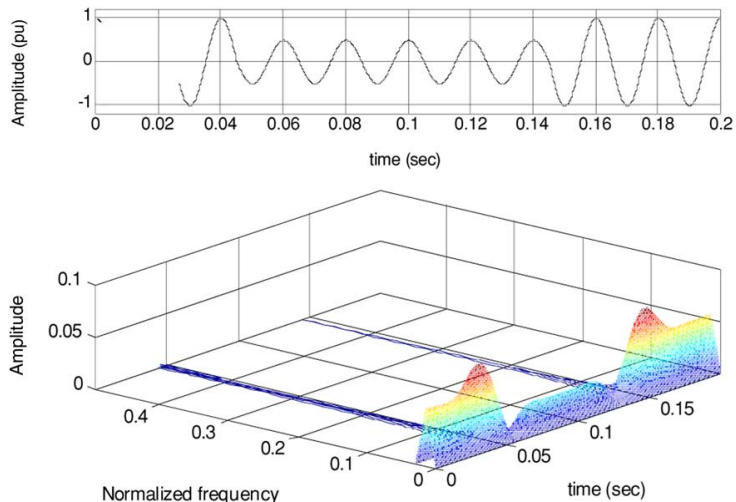

Fig. 4. ST spectrum of a sag event.
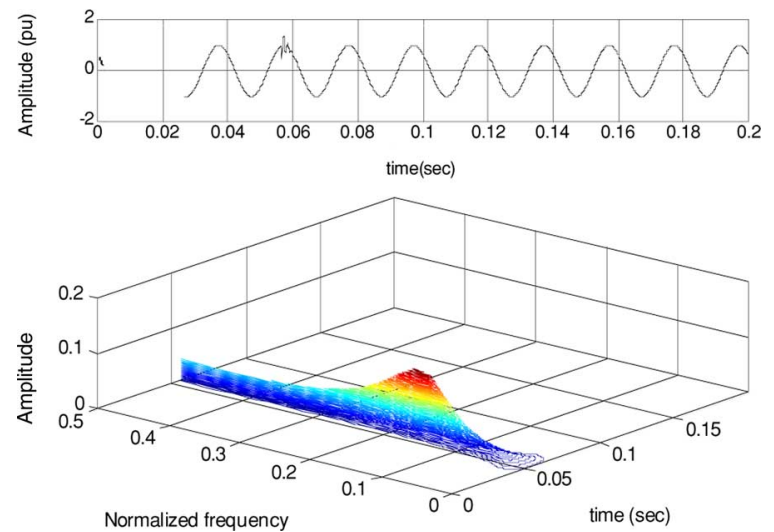

Fig. 5. ST spectrum of an oscillatory transient event.

The above equation represents a matrix, the rows of which are the frequencies, whereas the columns are the corresponding times. Each column, thus, represents the local spectrum at one point in time. Since the matrix $S[k T, n / N T]$ preserves the amplitude information of the frequency content of the signal at different resolutions, it can localize the different classes of power quality events accurately.

The accuracy of the ST spectrum on localizing power quality signals can be illustrated in two case studies as shown in Figs. 4 and 5 . In both cases, the test signals were simulated using MATLAB. The number of samples per cycle in both cases was 50 , and their fundamental frequency was $50 \mathrm{~Hz}$. The first case in Fig. 4 (top) represents a 0.5 p.u. voltage sag event while the second case in Fig. 5 (top) represents an oscillatory transient event. The amplitudes of the ST spectra, [the $S$ matrix in (9)] are presented as three-dimensional plots as shown in Fig. 4 (bottom) for the sag event, and in Fig. 5 (bottom) for the oscillatory transient event to demonstrate the variations in the amplitudes of the instantaneous frequencies in the signals.

The three-dimensional plots depict the ability of the ST spectrum to track the changes in the low-frequency components in the signal (as in the case of the sag event), as well as the changes in the high-frequency components (as in the case of the transient event). Considering this feature of the ST, a power quality monitoring algorithm is proposed in this study as explained the following section. 


\section{Automatic Monitoring Algorithm}

The proposed algorithm for automatic power quality monitoring is based on Parseval's Theorem. This theorem states that the energy of a signal $v(t)$ remains the same whether it is computed in a signal domain (time) or in a transform domain (frequency) as given in [15]

$$
E_{\text {signal }}=\frac{1}{T} \int_{0}^{T}|v(t)|^{2} d t=\sum_{n=0}^{N}|V[n]|^{2}
$$

where $T$ and $N$ are the time period and the length of the signal, respectively, and $V[n]$ is the Fourier transform of the signal.

In the case of the ST, the raw signal is decomposed in terms of its frequencies, and thus, a set of decomposed signals at each of the instantaneous frequencies in the raw signal can be obtained from the ST matrix. Thus, based on Parseval's Theorem, the energy of a distorted signal can be given as

$$
E_{\mathrm{ST}}\left[\frac{n}{N T}\right]=\sum_{k=1}^{N}\left(S\left[k T, \frac{n}{N T}\right]\right)^{2}
$$

where $n=1 \ldots N / 2, N$ is the signal length, and $E_{\mathrm{ST}}[n / N T]$ is the energy vector of the instantaneous frequency at frequency $n / N T$.

Although the energy vector $E_{\mathrm{ST}}$ can contain the instantaneous frequencies starting from the lowest frequency (the dc component) up to one-half of the sampling rate of the signal (Nyquist rule) at 1-Hz frequency resolution, in this study, a frequency resolution of $25 \mathrm{~Hz}$ for the ST calculations was found to be an appropriate selection for the power quality classification technique presented in this paper. Note that the fundamental frequency of the signals is assumed to be $50 \mathrm{~Hz}$. It should be also noted that the energies of the decomposed signals are calculated from the absolute value of the ST matrix which differentiates them from the traditional FFT technique.

As it will be explained below, based on the vector $E_{\mathrm{ST}}$, the proposed algorithm has the advantage of performing two simultaneous tasks automatically: 1) classification of power quality events, and 2) characterization of the classified events by identifying more information, (e.g., the depth, duration, starting and ending times of the events), which is required for analyzing the power quality issues.

\section{A. Classification of Power Quality Events}

It is proposed to use the energy vector of the instantaneous frequencies to distinguish different types of power quality disturbances. The distinguishing capability of the proposed energy vector was tested using five common power quality events: sags, swells, interruptions, oscillatory transients, and harmonic distortions. Table I provides the modeling details of the studied five events showing the controlling parameter of each event. The energies of the instantaneous frequencies of these events, based on (11), are demonstrated in Figs. 6-9.

In Fig. 6, the energies of the instantaneous frequency vectors are plotted against different magnitudes of sag events varying from 0 p.u. (pure signal) to 1 p.u. (interruption). The lowest curve in the figure represents a pure signal whereas the upper curve represents an interruption event. The frequency is normalized for the sampling rate of $5 \mathrm{kHz}$. From the figure, one

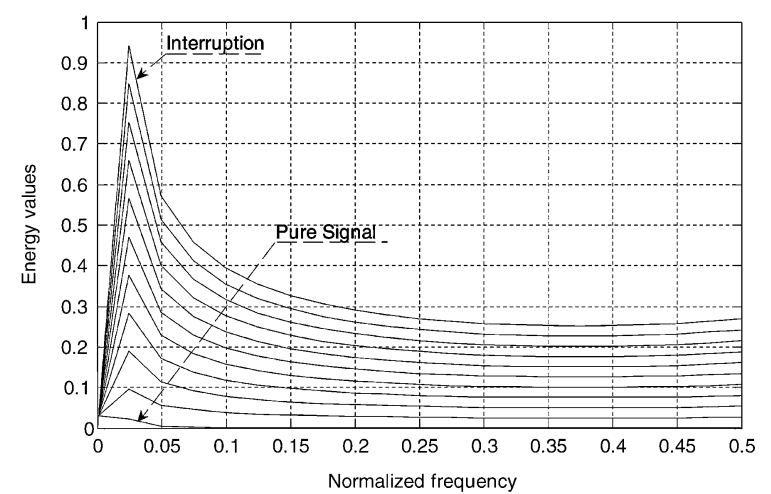

Fig. 6. Energies of the instantaneous frequencies of sag events of different magnitudes.

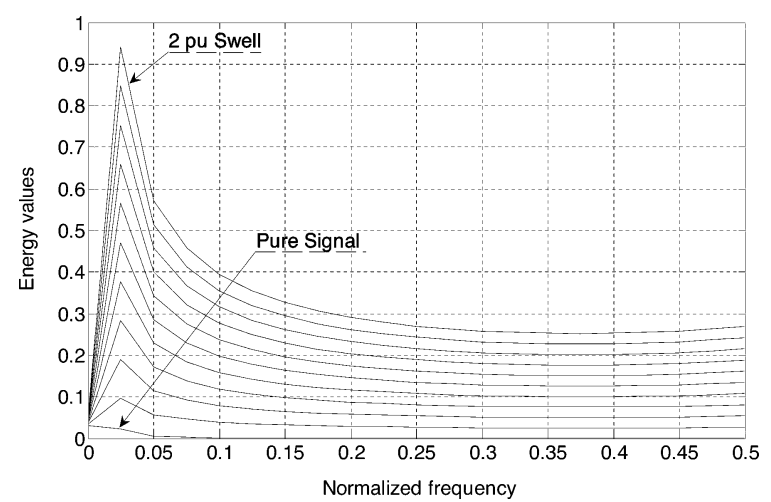

Fig. 7. Energies of the instantaneous frequencies of the swell events at different magnitudes.

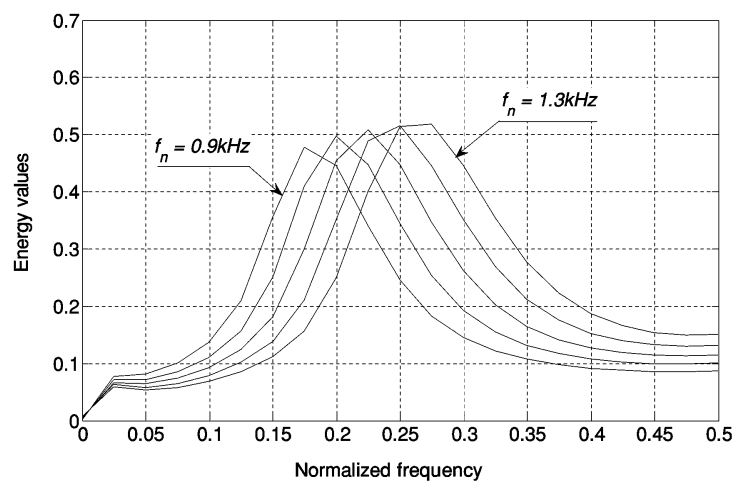

Fig. 8. Energies of the instantaneous frequencies of the transient events at different oscillation frequencies.

can see how the energies of the instantaneous frequency vector increases with the magnitudes of sag events.

The energies of the instantaneous frequency vectors in the case of swell events are shown in Fig. 7. Similarly, each curve in the figure illustrates the magnitudes of different swell events, which vary from 1 p.u., which represents a pure signal (the lowest curve in the figure), up to 2 p.u. swell (the highest curve in the figure). It should be noted that, although the profiles of the curves in this case are very similar to the sag events, there are slight differences in the minimum values of the curves as will be shown later in Fig. 12.

Fig. 8 is given to illustrate the energies of the instantaneous frequency curves in the case of oscillatory transient events. Five 
TABLE I

MODELING OF POWER QUALITY EvENTS

\begin{tabular}{l|l|l}
\hline Event Class & Modeling Equations & $\begin{array}{l}\text { Equations } \\
\text { Parameters }\end{array}$ \\
\hline \hline Pure Signal & $v(t)=A \cos (\omega t)$ & $\begin{array}{l}A \text { is Amplitude } \\
\omega=2 \pi f\end{array}$ \\
\hline Sag & $v(t)=A\left(1-k\left(u\left(t_{2}\right)-u\left(t_{1}\right)\right)\right) \cos (\omega t)$ & $\begin{array}{l}u(t) \text { is unit step } \\
\text { function, and } \\
0.1 \leq k \leq 0.9\end{array}$ \\
\hline Swell & $v(t)=A\left(1+k\left(u\left(t_{2}\right)-u\left(t_{1}\right)\right)\right) \cos (\omega t)$ & $0.1 \leq k \leq 0.9$ \\
\hline Interruption & $v(t)=A\left(1-\left(u\left(t_{2}\right)-u\left(t_{1}\right)\right)\right) \cos (\omega t)$ & \\
\hline & $v(t)=A \cos (\omega t)+h_{2} \cos (2 \omega t)+h_{3} \cos (3 \omega t)+$ & $\begin{array}{l}0 \leq h_{i} \leq 0.25 p u \\
\text { for: } i=3,5,7 \\
0 \leq h_{j} \leq 0.1 p u \\
\text { for: } j=2,9,11\end{array}$ \\
Harmonics & $h_{5} \cos (5 \omega t)+h_{7} \cos (7 \omega t)+h_{9} \cos (9 \omega t)$ & $\begin{array}{l}k=0.7 \\
\tau=0.0015\end{array}$ \\
& $h_{11} \cos (11 \omega t)$ & $\begin{array}{l}\omega_{n}=2 \pi f_{n} \\
900 \leq f_{n} \leq 1300\end{array}$ \\
\hline \multirow{2}{*}{ Transient } & $v(t)=A\left[\cos (\omega t)+k \exp \left(-\left(t-t_{1}\right) / \tau\right)\right.$. & \\
& $\left.\cos \left(\omega_{n}\left(t-t_{1}\right)\right)\left(u\left(t_{2}\right)-u\left(t_{1}\right)\right)\right]$ &
\end{tabular}

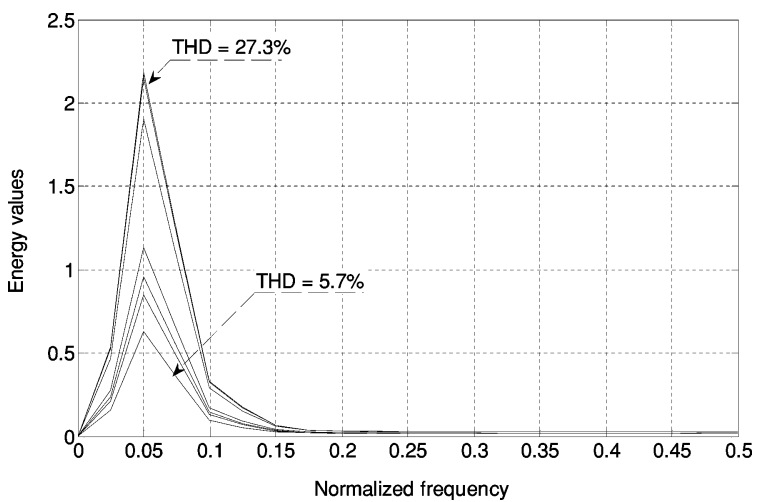

Fig. 9. Energies of the instantaneous frequencies of the harmonic distortion events at different values of THD.

oscillation frequencies of the events varying from 0.9 to $1.3 \mathrm{kHz}$ using the modeling equations given in Table I are shown in the figure.

The instantaneous frequencies' energies of the harmonic distortion events are shown in Fig. 9. In this case, the fundamental frequency was contaminated with the harmonics $2 \mathrm{nd}, 3 \mathrm{rd}$, 5th, 7 th, 9 th, and 11th. The magnitude of the harmonics is changed randomly between $0 \%$ to $25 \%$ of the fundamental for the $3 \mathrm{rd}$, 5 th, and 7 th, while the rest of the harmonics kept less than $10 \%$ as was shown in Table I. In Fig. 9, the corresponding curves of the energies of the instantaneous frequencies are shown for a selected ten different signals having a total harmonic distortion (THD) varied randomly from $5.7 \%$ to $27.3 \%$. The lower curve represents the instantaneous frequency's energies of a signal having a THD of $5.7 \%$.

From the figures given above, one can recognize three different frequency regions which can be used to distinguish the five power quality classes studied. These regions are illustrated in Fig. 10. The first region (region 1 in the figure) is for the first three classes of events: sag, swell, or interruption. Note that further analysis will be performed later in the paper to distinguish the three classes of events present in region 1 . The second region (region 2 in the figure) is related to the harmonic distortion

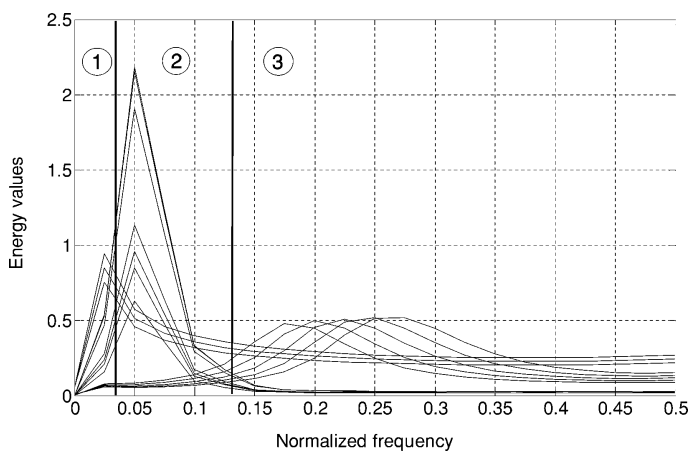

Fig. 10. Classification regions as specified by the proposed technique.

events, which usually having harmonics in the range of hundreds of hertz. The third region (region 3 in the figure) is for the oscillatory transient events which generally show much higher frequency components, (in the kilohertz range).

Based on Fig. 10, the power quality events can be classified using the proposed algorithm by calculating the maximum value of the energies of the instantaneous frequencies. If the maximum value lies in region 1, then the event could be either a sag or a swell, or an interruption. Similarly, if the maximum value lies in regions 2 or 3 , then the event is a harmonic distortion event or an oscillatory transient event respectively.

\section{B. Characterizing Power Quality Events}

Once the events are classified as explained above, further information about the characteristics of an event is usually required. This information is important to determine the cause of the event.

The information that can be extracted from an event depends on the class of the disturbance. For example, for the sag, the swell, and the interruption classes, the information required is the magnitude and the duration of the event, whereas the THD of the event is usually required in order to characterize the harmonic distortion events. In addition, to identify the source of the disturbance in the oscillatory transient class, it is helpful to know the starting time and the frequency of the oscillation. The following subsections will explain the ability of the proposed technique to efficiently quantify the disturbances in a signal using the maximum and minimum values of the instantaneous frequencies' energy curves. As was shown above, the events will be divided into regions as follows.

1) Quantifying the Classes in Region 1: Although the proposed technique has similar responses to the classes of sag, swell, and interruption, one can distinguish a specific class by calculating the minimum and maximum values of the instantaneous frequencies' energy curves. As will be seen in the following figures, these maximum and minimum values can be used to identify the magnitudes of the sag and the swell events.

Figs. 11 and 12 show the minimum values and the maximum values of the energy curves given in Figs. 6 and 7, respectively.

Fig. 11 shows that the maximum value of the energies of the instantaneous frequencies versus the magnitude of the sag and the swell events are the same for both of them. Therefore, by using this curve, the depth of the sag or the height of the swell can be identified directly. 


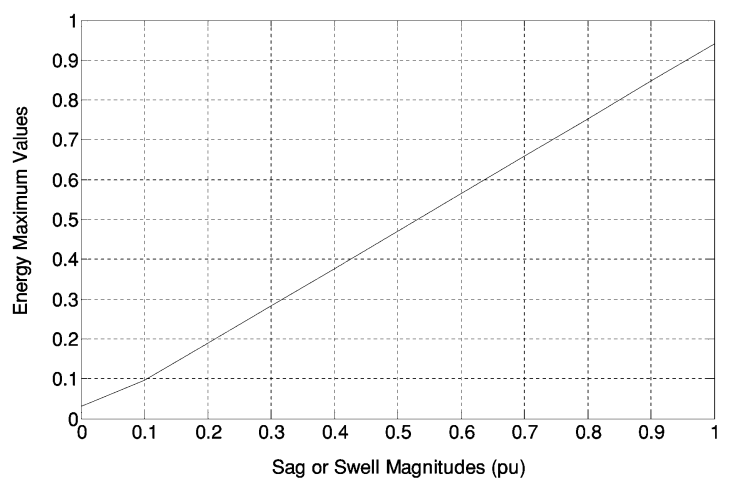

Fig. 11. Maximum values of the energies of the instantaneous frequencies for the sag and the swell events as a function of the magnitudes of these events.

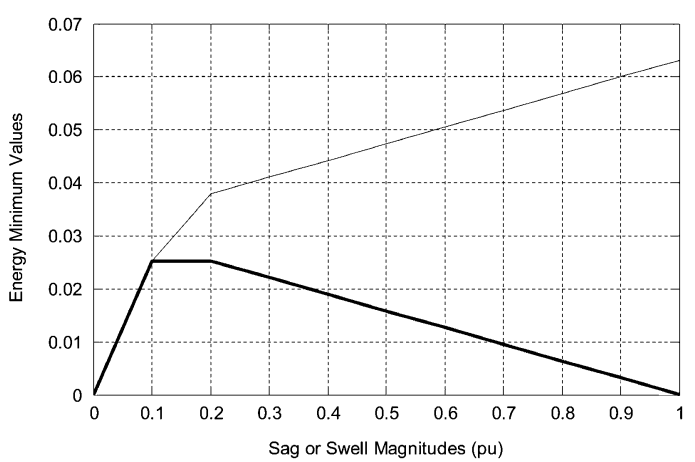

Fig. 12. Minimum values of the energies of the instantaneous frequencies for the sag (thick) and the swell (thin) events as a function of the magnitudes of these events.

The decision whether the event is a sag or a swell can be made from the minimum values of the energy curves as shown in Fig. 12. One can see in the figure that, if the minimum value of the energies of the instantaneous frequencies curve is less than 0.025 , then the event is a sag, otherwise it is a swell event. In the case of an interruption event, however, the identification can be made using both minimum and maximum values. If the maximum value is more than 0.9 and the minimum value of less than 0.003 , then the event is an interruption.

It should be noted here that there is an uncertainty about a decision if the magnitude of sag or swell events is less than 0.1 p.u., as can be seen in Fig. 12. However, this is not considered a limitation since the IEEE standards [16] still consider these small variations as pure (that is, acceptable) signals. Therefore, if the magnitude of an event is within this range, then the signal can be considered not distorted (pure signal), and hence requires no identification.

In order to identify the duration of an event, it was found that the signal symmetry can be utilized to identify the starting and the ending times of the event. This is because the occurrence of the event in a signal causes a sudden change in the symmetry of the signal. Such sudden changes usually involve a high-frequency component in the signal. Therefore, the time derivative of the energy of the highest frequency in the $S$ matrix defined by (9) can be used to detect the occurrence time(s) of the event. This can be given by

$$
t_{D}=\frac{d S[k T, 1 / 2 T]}{d(k T)}
$$

where $t_{D}$ is the disturbance occurrence time vector.
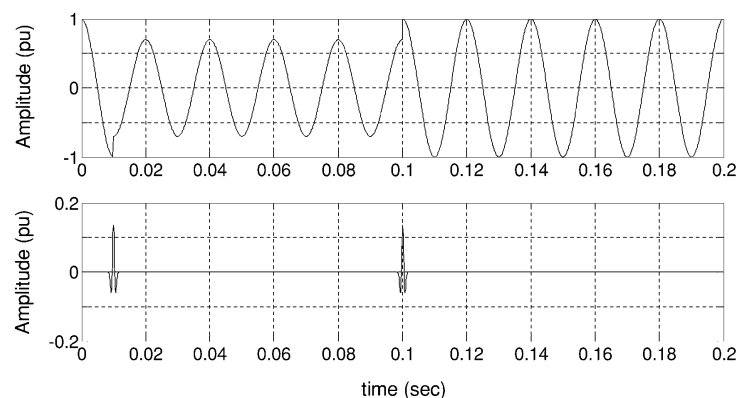

Fig. 13. Sag event of 0.3 p.u. (top) and its disturbance occurrence time vector (bottom).
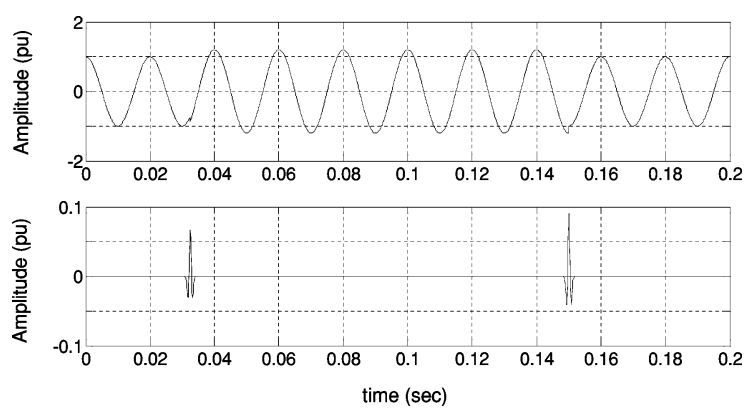

Fig. 14. Swell event of 0.2 p.u. (top) and its disturbance occurrence vector (bottom).

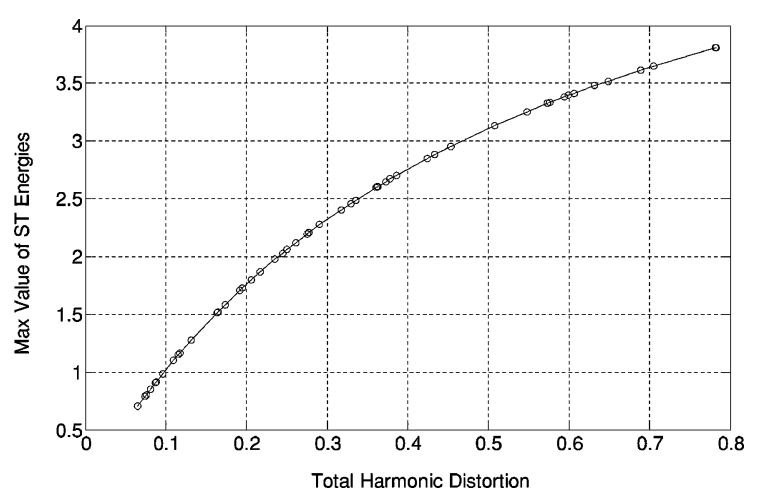

Fig. 15. The plot showing the maximum values of the energies of the instantaneous frequencies versus the total harmonic distortion of the signal.

The response of the $t_{D}$ vector is demonstrated in Figs. 13 and 14. Fig. 13 (top) shows that the voltage sag starts at $0.01 \mathrm{~s}$ and ends at $0.1 \mathrm{~s}$. These times are accurately detected by the $t_{D}$ vector, as shown in Fig. 13 (bottom).

Similarly, as shown in Fig. 14, the starting and ending times of a swell event of 0.2 p.u. was accurately detected by the disturbance occurrence times vector as 0.032 and $0.15 \mathrm{~s}$, respectively.

2) Quantifying the Classes in Region 2: If an event is classified in region 2 (which is a harmonic distortion event), then the THD should be specified for the event. In order to determine the THD directly using the proposed algorithm, the maximum values of the energies of the instantaneous frequencies are plotted against the THD as shown in Fig. 15. As can be seen from the plotted relationship between the two parameters, once the maximum value of the energies curves is detected, the THD can be determined accordingly. 

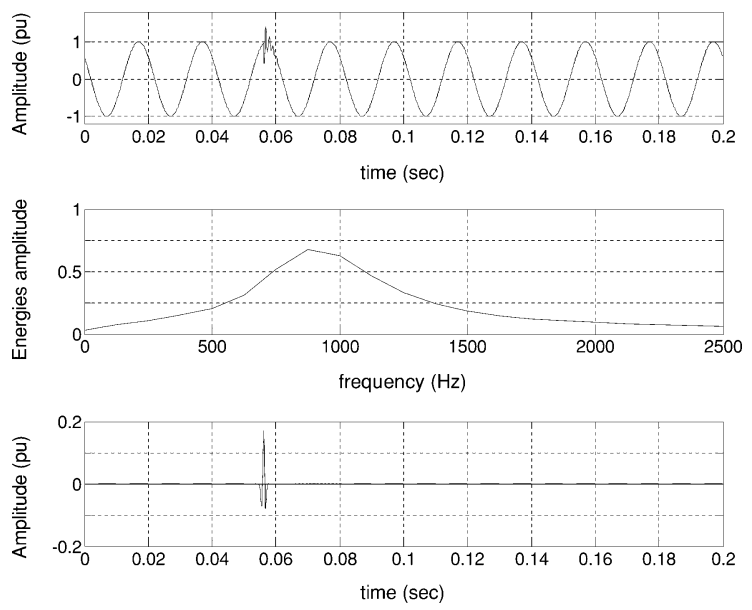

Fig. 16. Oscillatory transient event having (top), its energies showing an oscillation frequency of $900 \mathrm{~Hz}$ (middle) and corresponding disturbance occurrence vector (bottom).

3) Quantifying the Classes in Region 3: For events classified as region 3 events (oscillatory transients), the initial time and the oscillation frequency can be determined accurately using the proposed technique. In this case, the maximum value of the instantaneous frequency's energy curve corresponds to the frequency of oscillation.

This is illustrated in Fig. 16, which shows an oscillatory transient event having an oscillation frequency of $900 \mathrm{~Hz}$. The frequency of the superimposed oscillatory transient is detected accurately using the energies curve of the instantaneous frequencies (Fig. 16, middle). Similarly, the starting time of this event can be specified using the vector in (12), which is determined here as $0.056 \mathrm{~s}$.

The whole process of the proposed algorithm can be summarized in the flowchart shown in Fig. 17. This process will be examined in the following section using two case studies.

\section{ApPlication of the Proposed Algorithm}

In order to demonstrate the feasibility of the technique proposed above, different power quality events were generated using a power system simulation tool, which utilizes the SimPowerSystem Blockset of Simulink as a development platform. In the paragraphs below, two case studies are given to demonstrate the operation of the proposed algorithm.

The first case study represents a load fed from two transmission lines as shown in the single line diagram of Fig. 18 (top). In this configuration, if a fault occurs in one of the transmission lines, the load will experience a voltage sag until the fault is cleared. The simulation block diagram that investigates this case is given in Fig. 18 (bottom). In this study, it is assumed that the fault occurred at the midpoint of one of the transmission lines and lasted for seven cycles.

The voltage signals were captured at the load side at a sampling rate of $5 \mathrm{kHz}$. The voltage signal of one phase, the energies of the instantaneous frequencies of the event, and the disturbance occurrence time vector are all given in Fig. 19.

In Fig. 19 (middle), the maximum value of the energies of instantaneous frequencies was found as 0.76 . Because this value

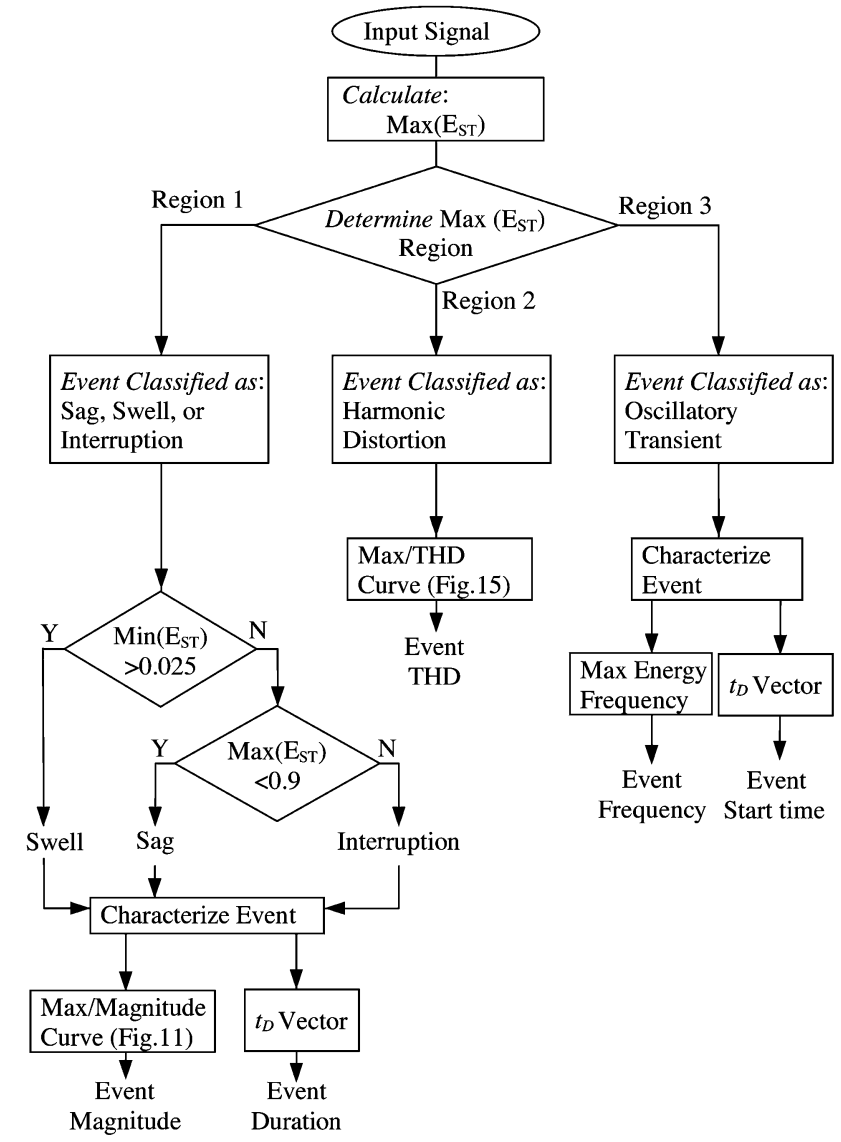

Fig. 17. Flowchart of the proposed classification and characterization algorithm.

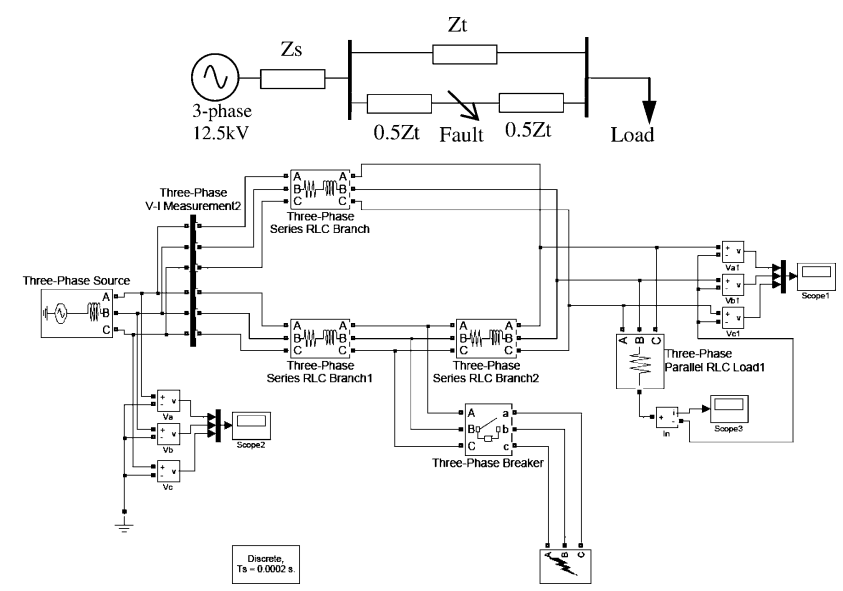

Fig. 18. Case study 1. A single line diagram of the computer simulation (top), which is used to generate a sag event, and its simulation block diagram based on SimPowerSystem Blockset of Simulink (bottom).

lies in the region 1 (as described in Fig. 10), the event is classified as a region 1 event, which could be either a sag, swell, or interruption event. However, since the minimum value of the curve of the energies of the instantaneous frequencies is 0.0073 , the event is classified as a sag event based on the curves provided in Fig. 12. To identify the magnitude of this sag event, the curve given in Fig. 11 can be used, which estimates the sag magnitude as 0.8 p.u., (which corresponds to a maximum energy value of 

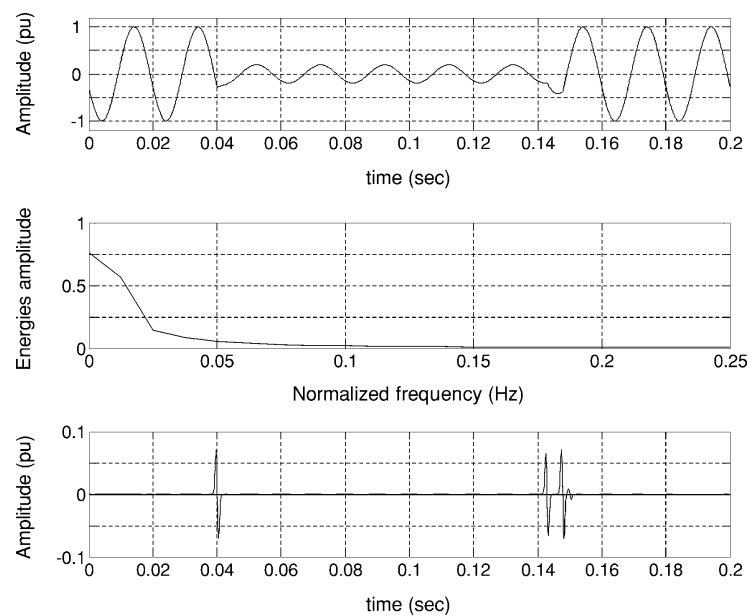

Fig. 19. Voltage sag due to a fault (top), its instantaneous frequencies energies curve (middle), and the disturbance occurrence times vector (bottom).
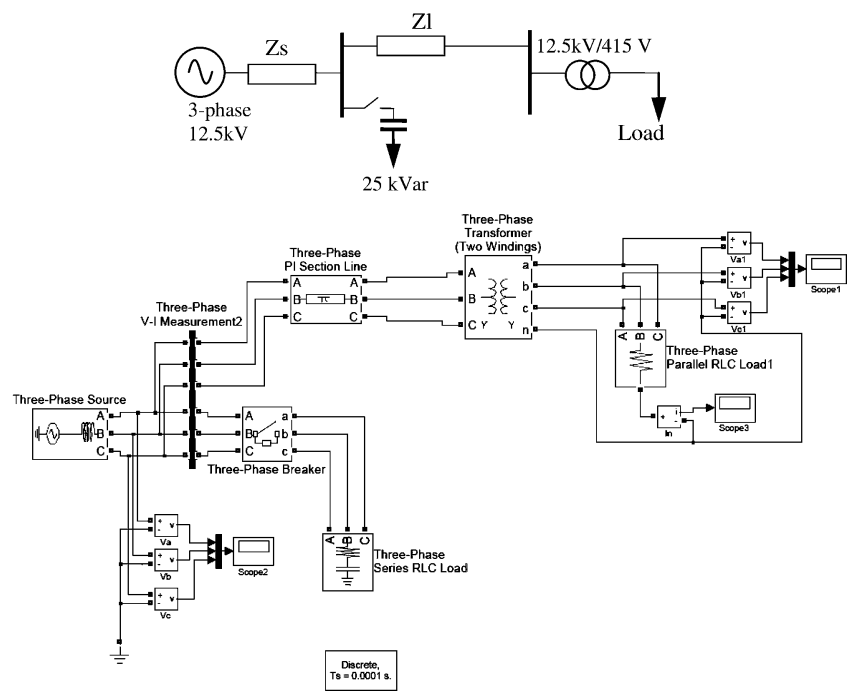

Fig. 20. Case study 2. Single line diagram of the (top) for the oscillatory transient event, and the simulation block diagram (bottom).

0.76). Note that, this estimated sag level is in agreement with a visual inspection of the sag signal in Fig. 19 (top). The second piece of information which can be obtained from the proposed algorithm is the starting and ending times of the event, and thus its duration. As it can be seen in Fig. 19 (bottom), the disturbance occurrence times vector detects three sudden changes in the signal: $0.0396,0.143$, and $0.148 \mathrm{~s}$. From a close inspection of the time-domain of the voltage signal, it can be seen that the recovery from the sag was in two stages, at 0.143 and $0.147 \mathrm{~s}$, which are accurately detected by the proposed algorithm.

The second case study examines the transient events that result from the connection of large capacitor banks to the supply in order to improve the power factor of the system. The single line diagram of this case study and its simulation block diagram are shown in Fig. 20.

In this case study, the capacitor banks are connected to the $12.5 \mathrm{kV}$ side and the voltage signals are captured at the $415 \mathrm{~V}$ side of the transformer (the load side) at a sampling rate of
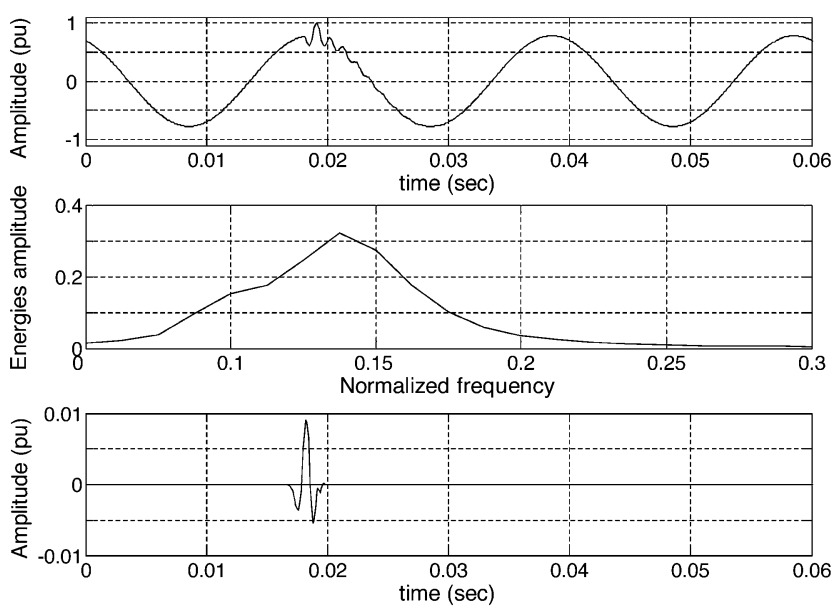

Fig. 21. Voltage transient due to the capacitor switching (top), its energies of the instantaneous frequencies (middle), and the disturbance occurrence time vector (bottom).

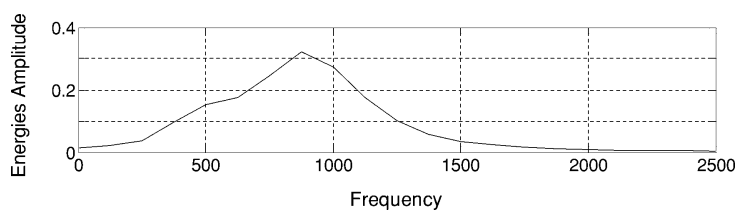

Fig. 22. Energies of the instantaneous frequencies of the oscillation transient event versus the actual frequency.

$5 \mathrm{kHz}$. The voltage signal of one phase, its energies of the instantaneous frequencies, and the disturbance occurrence time vector are shown in Fig. 21.

In Fig. 21 (middle), the maximum value of the energies of the instantaneous frequencies is estimated as 0.32 . Similar to the previous discussions, since this value lies in region 3 (see Fig. 10); therefore, it is an oscillatory transient event. Again, the starting time of this event was accurately detected by the disturbance occurrence time vector as $0.018 \mathrm{~s}$, as shown in Fig. 21 (bottom). The frequency of the oscillation can be identified as the frequency corresponding to the maximum value of the energy. To obtain the frequency of oscillation, Fig. 22 is given. In this figure, the instantaneous frequency energies curve is reproduced as a function of the actual frequencies, which indicates the frequency of the oscillation transient as $875 \mathrm{~Hz}$.

\section{CONCLUSION}

Due to the large number of power quality events that may occur in power systems, there is a need for a new technique that can be used for automated classification and characterization of power quality events. It is desirable that such a technique be able to both distinguish the type of power quality event, and also accurately identify the magnitude, duration, and frequency content of the disturbances.

This paper introduced a new algorithm to address the above requirements. The technique is based on the multiresolution S-transform. Unlike most of the previous automatic monitoring techniques that can only classify the disturbance type, the proposed technique has the ability to classify and also characterize the disturbances in the signals measured. 
In this paper, the energy vector from the multiresolution S-transform was used to distinguish the different types of power quality disturbances, which include five very common power quality events: sags, swells, interruptions, oscillatory transients, and harmonic distortion.

The classification and characterization of the disturbances in this proposed algorithm is based on simple rules using the energies of the instantaneous frequencies vector. The proposed algorithm was implemented for accurately distinguishing the five different power quality classes based on classification regions. It was demonstrated that the algorithm can efficiently quantify the disturbances in a signal using the maximum and minimum values of the variation of energy with instantaneous frequency.

In this paper, the performance of proposed approach was tested using two typical case studies based on simulations. It was demonstrated by the results that the technique is effective and accurate for both classification and characterizing of power quality events.

\section{REFERENCES}

[1] I. Y. Gu and E. Styvaktakis, "Bridge the gap: Signal processing for power quality applications," Electric Power Syst. Res., vol. 66, no. 1, pp. 83-96, Jul. 2003.

[2] W. R. A. Ibrahim and M. M. Morcos, "Artificial intelligence and advanced mathematical tools for power quality applications: A survey," IEEE Trans. Power Del., vol. 17, no. 2, pp. 668-673, Apr. 2002.

[3] R. A. Flores, "State of the art in the classification of power quality events, an overview," in Proc. 10th Int. Conf. Harmonics Quality of Power, 2002, vol. 1, pp. 17-20.

[4] Y. H. Gu and M. H. J. Bollen, "Time-frequency and time-scale domain analysis of voltage disturbances," IEEE Trans. Power Del., vol. 15, no. 4, pp. 1279-1284, Oct. 2000.

[5] A. M. Gargoom, N. Ertugrul, and W. L. Soong, "A comparative study on effective signal processing tools for power quality monitoring," in Proc. 11th Eur. Conf. Power Electron. Appl. (EPE), Dresden, Germany, Sep. 11-14, 2005, paper ID 915 (CD-ROM).

[6] F. Jurado, N. Acero, and B. Ogayar, "Application of signal processing tools for power quality analysis," in Proc. Can. Conf. Elect. Comput. Eng, May 2002, vol. 1, pp. 82-87.

[7] O. Poisson, P. Rioual, and M. Meunier, "Detection and measurement of power quality disturbances using wavelet transform," IEEE Trans. Power Del., vol. 15, no. 3, pp. 1039-1044, Jul. 2000.

[8] A. M. Gaouda, M. M. A. Salama, M. R. Sultan, and A. Y. Chikhani, "Application of multiresolution signal decomposition for monitoring short-duration variations in distribution systems," IEEE Trans. Power Del., vol. 15, no. 2, pp. 478-485, Apr. 2000.

[9] A. M. Gaouda, S. H. Kanoun, M. M. A. Salama, and A. Y. Chikhani, "Pattern recognition applications for power system disturbance classification," IEEE Trans. Power Del., vol. 17, no. 3, pp. 677-683, Jul. 2002.

[10] Z.-L. Gaing, "Wavelet-based neural network for power disturbance recognition and classification," IEEE Trans. Power Del., vol. 19, no. 4, pp. $1560-1568$, Oct. 2004.

[11] A. M. Gargoom, N. Ertugrul, and W. L. Soong, "Comparative study of using different mother wavelets on power quality monitoring," in Proc. Austral. Univ. Power Eng. Conf. AUPEC04, Brisbane, Australia, Sep. 26-29, 2004, paper ID 96 (CD-ROM).
[12] A. M. Gargoom, N. Ertugrul, and W. L. Soong, "A comparative study on effective signal processing tools for optimum feature selection in automatic power quality events clustering," in Proc. Ind. Appl. Conf. 40th IAS Annu. Meeting, Oct. 2-6, 2005, vol. 1, pp. 52-58.

[13] M. V. Chilukuri and P. K. Dash, "Multiresolution S-transform-based fuzzy recognition system for power quality events," IEEE Trans. Power Del., vol. 19, no. 1, pp. 323-330, Jan. 2004.

[14] R. G. Stockwell, L. Mansinha, and R. P. Lowe, "Localization of the complex spectrum: The S-transform," IEEE Trans. Signal Process., vol. 44, no. 4, pp. 998-1001, Apr. 1996.

[15] S. Haykin and B. Van Veen, Signals and Systems, 2nd ed. New York: Wiley, 2002.

[16] IEEE Recommended Practice for Monitoring Electric Power Quality, IEEE Std. 1159-1995, 1995.

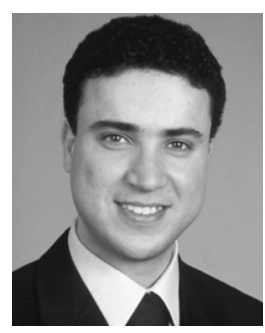

Ameen M. Gargoom (S'05) received the B.Sc. and M.Sc. degrees in electrical and electronic engineering from the University of Garyounis, Benghazi, Libya, in 1994 and 2001, respectively, and the Ph.D. degree from the University of Adelaide, Adelaide, Australia, in 2007.

From 1995 to 2001, he was a Consultant Engineer with AL-Emara Company in Libya before he joined the University of Garyounis as an Associate Lecturer. His main research interest includes power quality monitoring and signal processing technique applications in power systems.

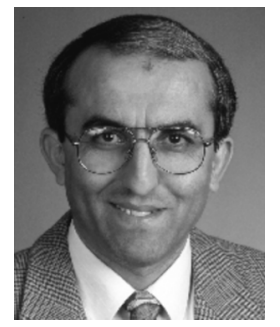

Nesimi Ertugrul (M'95) received the B.Sc. degree in electrical engineering and the M.Sc. degree in electronic and communication engineering from the Istanbul Technical University, Instanbul, Turkey, in 1985 and 1989 respectively, and the Ph.D. degree from the University of Newcastle, Nerwcastle upon Tyne, U.K., in 1993.

He has been with Adelaide University since 1994, where he is an Associate Professor. His primary research topics include sesnsorless operation of switched machines, power electronics, renewable energy systems, fault-tolerant motor drives, power quality monitoring, and condition monitoring and electric vehicles. He is the author of a book, LabVIEW for Electric Circuits, Machines, Drives, and Laboratories (Prentice-Hall, 2002). He serves on the Editorial Advisory Boards for the International Journal of Engineering Education (IJEE) and Journal of Power and Energy Conversion.

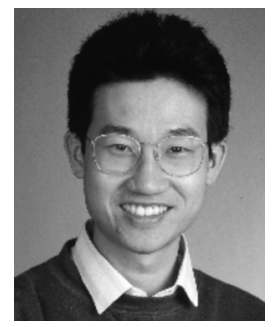

Wen L. Soong (M'93) was born in Kuala Lumpur, Malaysia. He received the B.Eng. degree from the University of Adelaide, Adelaide, Australia in 1989, and the Ph.D. degree from the University of Glasgow, Glasgow, U.K., in 1993.

He worked for four years as an electrical engineer in the Power Controls Program at General Electric Corporate Research and Development, Schenectady, NY, before taking up a teaching position in the Electrical and Electronic Engineering Department, University of Adelaide, in 1998. His present research interests include permanent magnet and reluctance machines, renewable energy generation, and condition monitoring and diagnostics. 